\title{
Carbonic Anhydrase Isoforms of Pyropia Yezoensis: Intracellular Location and Expression Profiles in Response to Inorganic Carbon Concentration and Life Stage
}

\author{
Baoyu Zhang \\ Institute of Oceanology \\ Xueying Liu \\ Ocean University of China \\ Li Huan \\ Chinese Academy of Sciences \\ Zhizhuo Shao \\ Chinese Academy of Sciences \\ Zhenbing Zheng \\ Chinese Academy of Sciences \\ Guangce Wang ( $\square$ gcwang@qdio.ac.cn ) \\ Institute of Oceanology
}

\section{Research Article}

Keywords: Carbonic anhydrase, subcellular localization; inorganic carbon utilization, Pyropia yezoensis

Posted Date: August 6th, 2021

DOI: https://doi.org/10.21203/rs.3.rs-737572/v1

License: (9) This work is licensed under a Creative Commons Attribution 4.0 International License. Read Full License 


\section{Abstract}

\section{Background}

Macroalgae, particularly commercially grown seaweed, substantially contribute to $\mathrm{CO}_{2}$ removal and carbon storage. However, knowledge regarding the $\mathrm{CO}_{2}$ concentrating mechanism (CCM) of macroalgae is limited. Carbonic anhydrase (CA), the key component of biophysical CCM, plays important roles in many physiological reactions in various organisms. CA has been widely studied in microalgae and higher plants. However, few characteristics of CA in Pyropia yezoensis are known, particularly its intracellular location and responses to different concentrations of $\mathrm{Ci}$.

\section{Results}

We retrieved transcriptomic and genomic data for $P$. yezoensis and identified, amplified and characterized 11 putative genes encoding CA. The predicted corresponding proteins clustered into three subfamilies: $\alpha$-, $\beta$ - and $\gamma$-type. Transcriptomic and qRT-PCR results revealed different expression levels of these PyCA genes in the two life stages of $P$. yezoensis, and their expression levels varied under different $\mathrm{Ci}$ conditions. The intracellular localization of eight CA isoforms-one in the chloroplasts, four in the cytoplasm and three in the mitochondriawere elucidated with fusion proteins. One yCA isoform (PyCA10) was shown to be localized in the cytosol, and its localization is discussed. We also discuss the potential roles of the CA isoforms in development and the CCM in the two life stages.

\section{Conclusions}

CA isoforms in P. yezoensis are widely distributed within cells, and higher PyCA expression, particularly of certain chloroplastic, cytosolic and mitochondrional CAs, is observed more often during the leafy stage. This expression results in stronger carbon sequestration ability in leafy thalli than filamentous thalli.

\section{Background}

Mitigation of climate change by decreasing $\mathrm{CO}_{2}$ emissions is urgently needed. Oceans are the largest $\mathrm{C}$ pool on Earth, and approximately $93 \%$ of global $\mathrm{CO}_{2}$ is contained within by the ocean [1]. Although oceanic carbon fluxes are believed to be dominated by microorganisms, marine vegetation, particularly commercially grown seaweed, also considerably contributes to $\mathrm{CO}_{2}$ removal and carbon storage $[2,3,4]$. Although substantial research on carbon sequestration by microalgae has been conducted, studies on carbon sequestration by multicellular macroalgae are relatively rare, particularly in cultured algae.

Pyropia yezoensis, a marine red alga, is an important marine crop widely grown through aquaculture in East Asia, such as in China, Japan and South Korea. The annual production of Pyropia is approximately 1,611,000 metric tonnes wet weight (data from FAO 2003). The life cycle of $P$. yezoensis mainly comprises a macroscopic leafy thallus phase (gametophyte) and a microscopic filamentous conchocelis phase (sporophyte), which alter each other. The artificial cultivated part of $P$. yezoensis is its leafy thalli. Because of its economic and ecological importance, and its research value, $P$. yezoensis is becoming a model macroalga among the Rhodophyta $[5,6]$.

To adapt to the low $\mathrm{CO}_{2}$ concentrations in seawater, Pyropia have evolved a $\mathrm{CO}_{2}$ concentrating mechanism (CCM) [7, 8]. Our previous research has indicated that $P$. yezoensis possesses biophysical CCM and biochemical CCM capabilities [9]. Carbonic anhydrase (CA), the key component of biophysical CCM, catalyzes the reversible dehydration of $\mathrm{HCO}_{3}{ }^{-}$to $\mathrm{CO}_{2}$, and also plays key roles in photosynthesis and respiration in many organisms $[10,11,12]$. With the development of sequencing technology, new types of CAs are increasingly being identified, and eight types of CA denoted with Greek letters a to $\theta$ have been reported $[13,14,15]$.

$\mathrm{CA}$ is widely distributed in compartments within the cells of plants, animals and microalgae [16,17]. Less information on CAs is available in macroalgae than in microalgae and higher plants, particularly regarding their functions and subcellular localization. To date, $\alpha$-, $\beta$ - and $\gamma$-type CAs have been found in Rhodophytes and Phaeophytea $[18,19,20]$. On the basis of the availability of the Pyropia genome, Wang and colleagues have reported that there are 24 and 22 putative CA homologs in $P$. yezoensis and $P$. haitanensis, respectively, all belonging to the $\alpha-, \beta$ - and $\gamma C A$ families [21]. Physiological reactions to enhanced $\mathrm{CO}_{2}$ or inhibitory experiments have indicated that $C A s$ play an important part in inorganic carbon acquisition in Pyropia [22, 23]; however, the exact functions and the distribution of individual CAs in Pyropia remain poorly characterized. To date, only one report has documented the subcellular localization of four PyCAs transiently expressed in heterologous tobacco epidermal cells [21]. Much effort is needed to elucidate the distribution and physiological roles of CA isoforms in Pyropia. 
In this work, $11 \mathrm{CA}$ genes were identified from the transcriptomic data and genome sequences of $P$. yezoensis. We studied the characteristics and subcellular localization of CA isoforms within the cell as well as changes in RNA expression under different Ci concentrations at two life stages. This work provides information that should aid in exploring the functions of CAs and in further analyzing the CCM of $P$. yezoensis.

\section{Results}

\section{Identification and analysis of CA genes}

We identified 11 CA genes from the $P$. yezoensis transcriptome and amplified them from genomic DNA using specific primers (S1 TableS1). Four CA genes belonged to the a type, three belonged to the $y$ type and four belonged to the $\beta$ type, including one previously reported $\beta C A$ renamed PyCA1. The characteristics of the other CA genes, the putative amino acid sequences and the distribution among chromosomes are listed in Table 1. Sequences of these CA genes have been submitted to GenBank with the accession numbers listed in Table 1.

Table 1

The characteristic of all the CA genes of Pyropia yezoensis

\begin{tabular}{llllllllllllll}
\hline name & & $\begin{array}{l}\text { accession } \\
\text { No. in NCBI }\end{array}$ & length & ORF & 5'UTR & 3'UTR & $\begin{array}{l}\text { amino } \\
\text { acids } \\
\text { number }\end{array}$ & $\begin{array}{l}\text { MW } \\
\text { (kD) }\end{array}$ & PI & subtype & $\begin{array}{l}\text { location in } \\
\text { chromosome }\end{array}$ \\
\hline PyCA1 & DN38784_c0_g1 & FJ160292 & 1153 & 825 & 177 & 151 & 274 & 29.8 & 8.51 & $\beta$ & C1 \\
\hline PyCA2 & DN99529_c0_g1 & MN132853 & 1867 & 693 & 876 & 298 & 274 & 29.8 & 11.29 & $\beta$ & C3 \\
\hline PyCA3 & DN126328_c0_g1 & MN132854 & 900 & 828 & 12 & 60 & 275 & 27.75 & 5.5 & a & C2 \\
\hline PyCA4 & DN84778_c0_g1 & MN132855 & 1503 & 591 & 770 & 142 & 225 & 23.65 & 4.78 & a & C1 \\
\hline PyCA5 & DN105259_c0_g1 & MN132856 & 1059 & 855 & 204 & 0 & 348 & 34.31 & 8.33 & $\beta$ & C2 \\
\hline PyCA6 & DN50495_c0_g1 & MN132857 & 1265 & 963 & 257 & 45 & 320 & 33.92 & 9.41 & a & C3 \\
\hline PyCA7 & DN91854_c1_g1 & MT375595 & 1192 & 693 & 308 & 191 & 229 & 22.81 & 5.37 & $\beta$ & C3 \\
\hline PyCA8 & DN91438_c0_g1 & MT375597 & 879 & 609 & 118 & 152 & 202 & 21.47 & 6.03 & a & C1 \\
\hline PyCA9 & DN92894_c0_g1 & MT375598 & 1222 & 873 & 185 & 164 & 290 & 29.02 & 4.71 & Y & C1 \\
\hline PyCA10 & DN146474_c0_g1 & MT375699 & 776 & 732 & 23 & 21 & 243 & 26.33 & 6.08 & Y & unknown \\
\hline PyCA11 & DN104395_c0_g1 & MN800877 & 1094 & 822 & 76 & 196 & 273 & 26.63 & 4.93 & Y & C2
\end{tabular}

\section{BCAs}

PyCA1, PyCA2, PyCA5 and PyCA7 are representative $\beta C A$ in $P$. yezoensis. The PyCA1 (BCA1) and PyCA5 (BCA3) genes have similar structures, consisting of three exons (S2 Fig. S1). The second exon comprises 95 nucleotides, with $86.3 \%$ similarity between genes. The two $\beta C A s$ share $60 \%$ and $41.5 \%$ nucleotide and amino acid identity, respectively (S3 Fig S2), and are found on chromosome 1 (C1) and chromosome 2 (C2), respectively (Table 1). Multiple transmembrane sites in PyCA5 were predicted by TMpred. PyCA2 ( 3 CA2) and PyCA7 ( $\beta C A 4)$ are composed of one exon; moreover, the open reading frame (ORF) of the two genes comprises 693 nucleotides (S2 Fig. S1), with more than 70\% identity at both the nucleotide level and the amino acid level (S3 Fig S2). These two genes are located on chromosome 3 (C3), with PyCA2 only $1.4 \mathrm{~kb}$ away from PyCA7, possibly because of a recent gene duplication. There is no transmembrane site in PyCA2, whereas the transmembrane site in PyCA7 spans from amino acids 124 to 141.

The zinc binding sites and active sites in the four $\beta C A$ s are all conserved with respect to those of reported $\beta C A$ f from other bacterial, microalgal and higher plant species, and are coordinated by two Cys residues and one His residue (Fig. 1A) [24, 25]. The active site conservation supports a role of these proteins in $\mathrm{CO}_{2}$ and bicarbonate conversion in different parts of $P$. yezoensis.

We generated a phylogenetic tree of the CAs by using the maximum-likelihood method, on the basis of the full-length ORFs of CA proteins (Fig. 2). In the phylogenetic tree, the four $\beta C A s$ are divided into two subclades. PyCA2 and PyCA7 clustered closely with CAs from $P$. haitanensis. Meanwhile, PyCA1 and PyCA5 cluster closely with CAs from rhodophytes and green algae, such as Porphyra umbilicalis, Porphyridium purpureum and Coccomyxa sp., thus indicating the different evolution and origins of these four $\beta C A s$. 


\section{aCAs}

PyCA3 (aCA1), PyCA4 (aCA2), PyCA6 (aCA3) and PyCA8 (aCA4) are representatives of aCAs in P. yezoensis, and their genes are all composed of one exon. These CAs are quite different from one another at the amino acid level, with less than $40 \%$ identity among them; the similarity at the nucleotide level is 17-38\% (S3 Fig S2).) These four aCAs also show low similarity with other reported PyaCAs at the amino acid and nucleotide levels, with the exception of PyCA8 and aCA9 having about 65\% identity. PyCA4 and PyCA8 are located on the same chromosome, C1. PyCA3 and PyCA6 are located on chromosomes $\mathrm{C} 2$ and C3, respectively (Table 1).

The four aCAs all have transmembrane sites, and PyCA3 has three transmembrane sites predicted by the TMpred server. The zinc binding sites of PyCA3 and PyCA4 differ from those of most reported aCAs, which have three His residues coordinating the Zn ion [25]. However, in PyCA3, Gln149 is substituted for the last conserved His residue, and PyCA4 lacks His at two conserved positions, which are replaced by Gln residues (Fig. 1B). This site structure was also observed in the aCAs of $P$. tricornutum; Although some PtaCAs lack the conserved His residues, these sequences have all been suggested to possess potential Zn ligands within the putative active domain [26].

Three PyaCAs (PyCA3, PyCA4 and PyCA6) and CAs from $P$. haitanensis and $P$. umbilicalis form a group in the phylogenetic tree, separated from the CAs from Arabidopsis thaliana, Chlamydomonas reinhardtii and Dunaliella salina. However, PyCA8 is separated from other PyCAs and closer to CA from A. thaliana in the phylogenetic tree (Fig. 2). These results indicate divergence in origin and evolution.

\section{YCAs}

There are three yCAs in P. yezoensis, in agreement with the report from Wang et al. (2020) [21]. PyCA9 (yCA1) and PyCA11 (yCA3) are located on chromosomes $\mathrm{C} 1$ and $\mathrm{C} 2$, respectively, while PyCA10 ( $Y C A 2$ ) could not be located on any of the chromosomes (Table 1). PyCA9 and PyCA10 are composed of one exon, whereas PyCA11 has three exons (S2 Fig.S1). The similarity of nucleotide sequences and amino acids sequences among the three YCAs in $P$. yezoensis is low, at approximately $30 \%$ and $15 \%$, respectively (S3 Fig S2).

According to the annotation of classical yCA from Methanosarcina thermophila (denoted as CAM), three histidine residues (His 115, His 151 and His 156) are essential for coordinating a $\mathrm{Zn}$ atom [31]. PyCA10 has two conserved His residues but not His151, which is replaced by glutamic acid (Fig. 1C). PyCA9 and PyCA11 lack the three conserved histidine residues and thus should be renamed as $\mathrm{Y}$-like CAs. According to the annotation of CAM, Arg93, Asp95, GIn109, Asp110, His151 and His156 are important residues in YCA that are predicted to function in proton transfer in the CAM mechanism [28]. Most residues corresponding to these residues are conserved in PyCA10, with the exception of GIn109 and His151, which are replaced by glycine and glutamic acid, respectively (Fig. 1C). The putative proton-transferring amino acids in CAM are mostly conserved in PyCA9 and PyCA11, which, unlike PyCA10, have transmembrane sites.

In the phylogenetic tree, PyCA10 and CA11 are clustered with CAs from bacteria and green algae or higher plants (Fig. 2). However, PyCA9 is distinct from PyCA10 and PyCA11 and clusters together with CAs from rhodophytes such as $P$. haitanensis, P. umbilicalis and Gracilariopsis chorda, thus suggesting a high degree of divergence in origin and evolution between PyCA9 and the other two YCAs.

\section{Intracellular localization}

We examined the subcellular localization of the 11 Pyropia CA proteins by using a combination of bioinformatic analysis and experimental localization of translational protein fusions. Prediction results from seven predictors are listed in S4 Table S2. The predictions from most programs were very similar for the PyBCAs PyCA1, PyCA2 and PyCA5, but not for PyCA7. Among the four PyaCAs, except for PyCA4, the prediction programs yielded inconsistent results. The three PyYCAs predictions from six programs nearly coincided. However, the results from YLoc and ChloroP contradicted those of the other predictors for most PyCAs. By combining the predictions from several predictors, we concluded that PyCA1 and PyCA6 are likely to be localized in the chloroplast, and PyCA3 has a signal peptide. PyCA9 and PyCA11 may be are localized in the mitochondria. PyCA2, PyCA4, PyCA5 and PyCA10 may be localized in the cytoplasm. The localization of PyCA7 and PyCA8 is unclear because the predictions did not agree with one another.

Experimental data highlight the limits of in silico predictions for analysis of subcellular localization. We introduced the plasmid PBWA (V) HS-PyCA-GLosgfp into Arabidopsis protoplasts by PEG4000 and used PBWA (V) HS- GLosgfp as a control.

We confirmed that the BCA PyCA1 was located exclusively in the chloroplast, because the GFP fluorescence overlapped with the red chlorophyll autofluorescence (Fig. 3A); the green fluorescence from GFP was dispersed evenly throughout the cytoplasm of each infected cell harboring the PBWA (V) HS - GFP control vector (Fig. 3I). PyCA2 is a cytoplasmic CA because the GFP fluorescence was observed predominantly in the cytoplasm, although GFP fluorescence was also observed in the nuclei of Arabidopsis protoplasts (Fig. 3B), in agreement with the prediction result. We did not observe GFP fluorescence in PyCA5 and PyCA7, and therefore were unable to define their location in Arabidopsis protoplasts. 
Combined GFP fluorescence and prediction results suggested that the aCA PyCA3 may be located in the endoplasmic reticulum (Fig. 3C). PyCA4 is also cytoplasmic, similarly to PyCA2 (Fig. 3D). The most interesting localization result was that of PyCA6, which did not appear to in chloroplast, as indicated by the green fluorescence of GFP (Fig. 3E), a finding contrary to the prediction. To verified its exact localization, we introduced the mitochondrial marker MSTP into the expression plasmid PBWA (V) HS-MSTP-GLosmkate. The green fluorescence from the targeted protein overlapped with the red fluorescence from the marker protein and showed yellow fluorescence, thus indicating that PyCA6 is mitochondrial (Fig. 4). GFP fluorescence was not observed in Arabidopsis protoplasts transformed with the PyCA8-GFP fusion.

The GFP fluorescence suggested that the $\gamma C A$ P PyCA9 and PyCA11 are located exclusively in the mitochondria (Fig. 3F and 3H), but PyCA10 is localized in the cytoplasm (Fig. 3G), in agreement with the prediction results (S4 Table S2).

\section{RNA expression differences between two life stages and under different $\mathbf{C i}$}

PyCA1, PyCA2, PyCA4, PyCA5 and PyCA6 correspond to DN38784_c0_g1, DN99529_c0_g1, DN84778_c0_g1, DN105259_c0_g1 and DN50495_c0_g1, respectively, and the transcript abundance of these five CA genes in response to different Ci concentrations during the two life stages of $P$. yezoensis has been demonstrated and discussed in previous work [9]. We therefore determined the transcript abundance of the remaining six CA genes under different $\mathrm{Ci}$ conditions during the two life stages by using transcriptomic data (Fig. 5A), and we verified these results by using real-time fluorescent quantitative reverse-transcription PCR (qRT-PCR) (Fig. 5B). Primers for qRT-PCR are listed in S5 Table S3.

The abundance of $P Y C A 3$ in filamentous thalli was so low that it was almost undetectable. However, the PyCA3 abundance was very high in leafy thalli, at a level more than 20-fold higher than that in filamentous thalli, as verified by qRT-PCR. In contrast, PyCA10 was expressed in filamentous thalli and was almost undetectable in leafy thalli. The abundance of PyCA10 was not very high, and the reads per kilobase of exon model per million mapped reads (RPKM) value was approximately 7.93 under normal inorganic carbon conditions (NC). The Ct value of PyCA10 from qRT-PCR in the two life stages was over 32, indicating that the RNA abundance of PyCA10 was low. The PyCA8 transcript abundance was very low during the two life stages, and the RPKM value was less than 1. PyCA7, PyCA9 and PyCA11 showed greater abundance of RNA during the two life stages; moreover, the RNA content of PyCA7 and PyCA9 in filamentous thalli was higher than that in leafy thalli, (approximately 6- and 1.7-fold higher, respectively). In contrast, the abundance of PyCA11 transcript in leafy thalli was approximately 1.6 -fold higher than that in filamentous thalli.

Transcriptome data indicated that low carbon conditions (LC) induced expression of PyCA3 and PyCA9 in leafy thalli, to levels approximately twofold higher than those under control carbon conditions (NC). No clear difference was observed between LC and NC in the filamentous thalli (Fig. 5A). Low carbon had clear effect on the expression of PyCA7 in the filamentous thalli, but no obvious effect on its expression in leafy thalli. High $\mathrm{Ci}$ concentrations $(\mathrm{HC})$ decreased the expression of PyCA7 in leafy thalli; no clear difference between $\mathrm{HC}$ and $\mathrm{NC}$ in filamentous thalli was observed. The PyCA8 gene was highly expressed at the transcript level in filamentous thalli under LC, as indicated by an RPKM approximately threefold higher than that under NC. Expression of PyCA9 was induced by LC but not HC, as compared with NC in leafy thalli; however, PyCA9 expression in filamentous thalli was induced by HC but not LC, as compared with NC. HC and LC had no clear effect on the expression of PyCA10 in filamentous thalli. LC and HC induced expression of PyCA11 at the two life stages but to a similar extent as NC.

qRT-PCR showed an expression tendency similar to that revealed by transcriptomic data, with the exception that PyCA10 expression under LC was higher to that under NC in filamentous thalli, but was similar to that under NC in the transcriptomic data.

\section{Discussion}

\section{Subcellular localization}

Determining the cellular localization of each protein is necessary and is critical to understanding the biological function of CAs in $P$. yezoensis. Although several protein predictors are publicly available online, they display poor consensus in their predictions for many protein sequences, such as gene family proteins. As shown in S4 Table S2, the program YLoc had the least similarity to the other five programs in terms of targeting prediction and was followed by ChloroP. However, the program YLoc was demonstrated to be efficient in processing highly hydrophilic late embryogenesis abundant (LEA) proteins of Arabidopsis [29].

Proteins belonging to the ancient carbonic anhydrase clades $\beta$ and $a$ are distributed in many compartments within the cells of microalgae and higher plants, such as plasma membrane, cytoplasm, mitochondria and chloroplast [11,30]. The PyßCA PyCA1 is clearly localized to the chloroplast according to our subcellular localization and prediction results (Fig. 3A and S4 Table S2). PyCA2 is localized to the cytosol, in agreement with the results for PyßCA4, the same gene, reported by Wang (2020) [21]. In our work, the a-CAs PyCA3, PyCA4 and PyCA6 showed different cellular localizations, whereas another three aCAs, aCA5, aCA9 and aCA14, have been reported to localize to the cell wall

Page 5/16 
[21]. These six aCAs share low similarity at the protein and nucleotide levels (S3 Fig.S2). The number of aCAs in $P$. yezoensis is higher than that in $C$. reinhardtii (three aCAs) and $P$. tricornutum (five aCAs), and similar to that in $A$. thaliana (eight aCAs) $[17,30,31]$. However, little information is available regarding the localization of aCAs with respect to $\beta C A s$ in microalgae and higher plants.

An interesting CA protein identified in this work is PyCA10, a yCA. To date, $y$ - or $y$-like CAs have been regarded as part of mitochondrial Complex I in plants and microalgae $[32,33,34,35]$. We found that PyCA9 and PyCA11, the two $y$-like CAs, are exclusively mitochondrial (Fig. 3F and $3 \mathrm{H}$ ). Interestingly, however, PyCA10 is localized to the cytosol although it is considered a yCA with respect to its conserved active sites, and it shares $44-45 \%$ sequence identity with CAs from Solanum pennellii and Papaver somniferum, which are predicted to localize to the mitochondria (GenBank accession no. XP_015067256 and XP_026435912, respectively). Many mitochondrial proteins are biosynthesized as precursors in the cytosol with an $\mathrm{N}$-terminal mitochondrial targeting sequence (MTS) that is cleaved during the import process. The MTS variability hinders robust sub-cellular location predictions in silico [36]. Neither transmembrane sites nor signal peptides in PyCA10 were indicated by TMpred, SignalP or iPSORT programs (S4 Table S2). We cannot be certain whether the coding region of PyCA10 gene is complete because it was not found in the genome sequence of $P$. yezoensis. Another reason for PyCA10 for confusion regarding PyCA10 localization may be due to MTS loss after duplication. Two LEA proteins in Arabidopsis, LEA38 and LEA2, are highly similar paralogs ( $71 \%$ identity in coding sequence). LEA38 is targeted to mitochondria while LEA2 is cytosolic, according to observations based on fluorescent protein [29]. Avelange-Macherel and colleagues have studied the subcellular localization of LEA38 and LEA2 by constructing mutants and have found that LEA2 is redirected to the mitochondria. The authors have suggested that the LEA2 gene lost its MTS after duplication [37]. Similarly, PyCA10 might potentially localize to the mitochondria.

For the cytosolic proteins, we observed green fluorescence in the nucleus as well as the cytoplasm (Fig. 3B, 3D). In fact, this phenomenon has also been observed for Arabidopsis proteins in native cells [29]. Candat has proposed two possible reasons for this observation: passive diffusion of fusion proteins into the nucleus may occur; alternatively, degraded target gene-GFP that has lost the nuclear localization sequence but retains fluorescent might be present [29]. Given the function of CAs, passive diffusion of fusion proteins appears to be a more reasonable explanation in our work.

Two main methods are currently used for elucidating the localization of target proteins in macroalgae: immunolocalization and fluorescent protein translational fusions. With the development of genetic transformation technology, overexpression plasmids carrying target genes have been widely applied in model plants, such as Nicotiana tabacum, Arabidopsis and C. reinhardtii. Owing to a lack of perfect genetic transformation technology in macroalgae, particularly for subcellular localization in native cells, heterologous localization should be a good choice. Bi et al. have identified a mitochondrial $y C A$ in Saccharina japonica by using immunogold localization and have verified its location by expressing SjCA::GFP plasmid in onion epidermis [19]. Wang et al. (2020) have defined the localization of four CAs by overexpressing GFP and target genes in tobacco cells [21]. During heterologous localization experiments, we found that, even with the same expression plasmid, genes may be expressed in Arabidopsis but not in tobacco, or vice-verse; green fluorescence from a PyCA7 fusion was weak in tobacco and undetectable in Arabidopsis protoplasts (data not shown). Thus, much work is necessary to choose an expression plasmid and receptor for applying the heterologous localization method to $P$. yezoensis proteins.

\section{CA isoform functions in CCM}

CA function is tightly associated with its localization in the cell. Several studies suggest that CAs in the chloroplast and cell wall or periplasmic space are essential for the CCM of cyanobacteria, microalgae and higher plants $[11,14,25]$. PyCA1 is localized exclusively in the chloroplast and shows higher transcript abundance during the two life stages, with a RPKM value under NC above 2000 and above 600 in leafy thalli and filamentous thalli, respectively. Furthermore, PyCA1 is up-regulated under LC conditions [9]. On the basis of its subcellular localization and transcript abundance, we suggest that PyCA1 plays important roles in the CCM of $P$. yezoensis during the two life stages.

Data from higher plants indicate that the function of cytosolic CA varies according to the inorganic carbon utilization pathway of the individual species. Single mutants of cytosolic $\beta C A 2$ and $\beta C A 4$ in Arabidopsis show growth rates indistinguishable from those of wild-type plants, whereas disrupting both $\beta C A 2$ and $\beta C A 4$ together results in not only slow growth, but also low aspartate content in mutant plants [38]. Cytoplasmic CAs in Flaveria bidentis, a representative $\mathrm{C} 4$ dicot, play a key role in converting $\mathrm{CO}_{2}$ to $\mathrm{HCO}_{3}{ }^{-}$for PEP carboxylase [39]. Although 19 and 13 CA genes have been identified in $C$. reinhardtii and P. tricornutum, respectively, research on cytosolic CA function in these two species is limited $[14,31]$. P. yezoensis is a representative of the old Rhodophyta, and the function of its cytosolic CA may be different from those in specific $\mathrm{C} 4$ plants and C3 plants. On the one hand, biophysical CCM and biochemical CCM exist in P.yezoensis, although information about biochemical CCM is limited [9]. On the other hand, the leafy thalli of $P$. yezoensis show active $\mathrm{HCO}_{3}{ }^{-}$uptake from seawater, besides uptake of atmospheric $\mathrm{CO}_{2}$, as indicated by physiological experiments and transcriptomic data $[23,40]$. All these results indicate that cytosolic CAs are important for the leafy thalli of $P$. yezoensis. Moreover, the RPKM of PyCA2 (DN99529_c0_g1) in leafy thalli is approximately 500, a value 10-fold higher than that in filamentous thalli [9]. PyCA3 was also well expressed in leafy thalli but poorly 
expressed in filamentous thalli, whereas PyCA4 was poorly expressed during both life stages [Fig. 5]. Therefore, we propose that PyCA2 and PyCA3 play important roles in CCM in leafy thalli.

The mitochondrial PyCAs, PyCA6 and three $ү C A s$, had relatively high transcript abundance, especially PyCA6, showing the second highest transcript abundance among the $11 \mathrm{CA}$ genes in leafy thalli at approximately half that of PyCA1 [9]. Previous work has shown that pyruvate carboxylase in mitochondria has high carboxylation activity, using $\mathrm{CO}_{2}$ to produce oxaloacetate (OAA), an important TCA cycle substrate [9]. Beyond phosphoenolpyruvate carboxykinase, other enzymes utilizing $\mathrm{HCO}_{3}{ }^{-}$in the mitochondrion include 3-methylcrotonyl-CoA carboxylase (MCC), which is involved in leucine catabolism [41, 42]. Leucine is an essential amino acid for animals and is abundant in P. yezoensis [43], thus indicating that PyCA6 is essential for the supply of substrate for mitochondrial metabolism in leafy thalli.

Research on YCAs in microalgae and plants is limited, and whether they are involved in the CCM is unclear. RNAi knockdown strains for $y C A s$ in Arabidopsis show diminished levels of Complex I and altered physiology [34]. Wei et al. (2017) have studied a predicted mitochondrial yCA in Nannochloropsis oceanica by using RNAi technology and reported that a mutant with down-regulated yCA showed significantly decreased cell growth under atmospheric $\mathrm{CO}_{2}$, which affected photosynthetic oxygen evolution. Thus, the authors have suggested that this $\gamma C A$ is likely to play a role in the CCM [44]. Because PyCA9 and PyCA11 showed higher transcript abundance in the two life stages and PyCA10 is expressed exclusively in filamentous thalli (Fig. 5), we propose that these three $\gamma C A$ s are necessary for the filamentous thalli, whereas PyCA9 and CA11 are important for leafy thalli. Combining the above experimental results and analysis of the eight PyCAs, we created a diagram of PyCA localization in P. yezoensis (Fig. 6).

Leafy thalli are distributed among intertidal rocks, where they experience dehydration for hours and subsequent rehydration with the change in tide, and the living condition is complicated and crucial for leafy thalli. Because filamentous thalli grow in a calcareous matrix within subtidal zone, their living conditions are simple and stable, but oligotrophic. Leafy thalli must maintain rapid growth while living in such complicated conditions. That requirement explains why so many CA genes are present in the genome of $P$. yezeonsis and why higher $P y C A$ expression is observed more often during the leafy stage, especially of some chloroplastic, cytosolic and mitochondrional CAs, which is also the main reason for the stronger carbon sequestration ability of leafy thalli than filamentous thalli.

\section{Conclusions}

There are large number of CA genes in $P$. yezoensis's genome and the intracellular localization of eight CA isoforms were elucidated with fusion proteins. Higher PyCA expression, especially of some chloroplastic, cytosolic and mitochondrional CAs, is observed more often during the leafy stage. This results in stronger carbon sequestration ability of leafy thalli than filamentous thalli.

\section{Materials And Methods}

\section{Algal materials and culture conditions}

The material used herein was similar to that reported previously [9].

\section{Identification and cloning of putative carbonic anhydrase genes in P. yezoensis}

Candidate CA genes were screened from the $P$. yezoensis transcriptome and genome database (https://db.cngb.org/cnsa/ with accession number CNP0000880; BioProject PRJNA589917 in NCBI) [9, 21]. Putative sequences were analyzed with NCBI BLASTX (https://blast.ncbi.nlm.nih.gov/Blast.cgi) to confirm inclusion of the conserved CA domain. Candidate genes were cloned as follows. Total RNA was extracted with an RNAprep pure plant kit (Tiangen Biotech Co., Beijing, China) according to the manufacturer's instructions and dissolved in diethyl pyrocarbonate (DEPC)-treated water. cDNA used for amplifying complete CA gene sequences was synthesized with Moloney Murine Leukemia Virus (MMLV) reverse transcriptase (Promega Biotech Co., Madison, WI, USA), and cDNA used for qRT-PCR was synthesized with a PrimeScript ${ }^{\text {tM }}$ RT reagent kit with gDNA Eraser (TaKaRa Bio, Beijing, China).

\section{Sequence analysis, phylogenetic tree reconstruction and prediction of subcellular localization}

Alignment and comparison of deduced protein sequences was performed with ClustalW [45], and transmembrane regions were predicted with the TMpred tool (http://www.ch.embnet. rg/software/TMPRED_form.html). A phylogenetic tree was reconstructed with the maximumlikelihood (ML) calculation in MEGA version 6 [46]; the model/method was WAG $+\mathrm{G}+\mathrm{l}$, and gaps/missing data treatment was set to use all sites. A total of 1,000 bootstrap replicates were performed. 
Bioinformatic predictions were made with the following publicly available subcellular targeting prediction programs: SignalP (http://www.cbs.dtu.dk/services/SignalP/), TargetP (http://www.cbs. dtu.dk/services/TargetP/), ChloroP

(http://www.cbs.dtu.dk/services/ChloroP/), Predotar (https://urgi.versailles.inra.fr/predotar/), iPSORT (https://ipsort.hgc.jp/index.html), MultiLoc2 (https://abi-services.informatik.uni-tuebingen.de/multiloc2/webloc.cgi) and YLoc (https://abi-services.informatik.unituebingen.de/yloc/webloc.cgi).

\section{Plasmid constructs for EGFP fusion and genetic transformation}

The complete coding regions of CA genes were amplified using primers containing Bsal restriction endonuclease sites (CGTCTC), and PCR products were purified from agarose gels. After digestion with Bsal endonuclease, PCR products were cloned into the vector PBWA (V) HSGLosgfp (Wuhan Biorun Biotech Co. Ltd., China) with T4 ligase assembly. Positive clones on medium containing kanamycin were confirmed by sequencing. The PyCA coding region lies on the N-terminus of the GFP. PyCA:GFP expression in transgenic plants was driven by the cauliflower mosaic virus $35 \mathrm{~S}$ constitutive promoter. The plasmid for expression of the Arabidopsis mitochondrial signal protein MSTP was constructed upstream of the red fluorescent protein MKATE according to the above-described method [47]. Transformation into Arabidopsis protoplasts was mediated by PEG4000 [48], and transformants were observed under laser-scanning confocal microscopy (Nikon C2-ER; Japan). Wavelengths of $480 \mathrm{~nm}$ and $640 \mathrm{~nm}$ were used for excitation of GFP and chlorophyll, respectively. Emission wavelengths of $510 \mathrm{~nm}$ and $675 \mathrm{~nm}$ were used for GFP and chlorophyll, respectively. MKATE was excited at $561 \mathrm{~nm}$ and detected at $580 \mathrm{~nm}$.

\section{qRT-PCR assay}

The relative expression levels of PyCA3, PyCA7-11 genes were measured by qRT-PCR. The protocol for qRT-PCR was performed with standard methods (Roche, Switzerland) as described previously [20], with some modifications, specifically that glyceraldehyde-3-phosphate dehydrogenase (GAPDH) of $P$. yezoensis was also applied as an internal reference gene in addition to Eukaryotic initiation factor 4A (EIF4A, TRINITY_DN83248_C0_g1). The results of qRT-PCR for the two housekeeping genes were similar. Primer pairs used for qRT-PCR analyses are listed in supplemental file 5, Table S3.

\section{Statistical analysis}

All the results in this study are shown as mean values \pm standard deviation $(n=3)$. The data were firstly analyzed using one-way ANOVA and then Tukey's test was used for post hoc analysis at the $p=0.05$ significance level. All analyses were carried out using SPSS 18.0 (SPSS Inc., Chicago, II, USA).

\section{Abbreviations}

CA: carbonic anhydrase; CCM: carbon concentration mechanism; GFP: green fluorescent protein; HC: high carbon; LC: low carbon; MCC: 3methylcrotonyl-CoA carboxylase; NC: normal carbon; RPKM: reads Per kilobase of exon model per million mapped reads.

\section{Declarations}

\section{Ethics approval and consent to participate}

Not applicable.

\section{Consent for publication}

Not applicable.

\section{Availability of data and materials}

All of the datasets supporting the results of this article are included within the article and its additional files. The raw reads of transcriptome in this study can be accessed with the link below: https://db.cngb.org/search/project/CNP0000880/, and public access to the database is open.

\section{Competing interests}

All the authors declare that they have no competing interests. 


\section{Funding}

This work was supported by the National Natural Science Foundation of China $(41876163,41876160,41776150)$, the National Key R\&D Program of China (2018YFD0901500), the Key Deployment Project of the Centre for Ocean Mega-Research of Science, the Chinese Academy of Sciences (COMS2019Q02), the Major Scientific and Technological Innovation Projects in Shandong Province (2019JZZY010815), and the North Jiangsu Science and Technology Special Project (LYG-SZ201916). These funding bodies had no role in study design, data collection, analysis, in writing the manuscript or the decision to submit the work for publication.

\section{Authors' contributions}

BZ conceived, designed research and amplified all the CA genes; $\mathrm{XL}$ and LH constructed the expression plasmid and transformed them into Arabidopsis protoplast. SZ cultivated the materials and did the QRT-PCR. ZZ observed the transformants under laser-scanning confocal microscopy. GW discussed the results and revised the manuscript. All authors read and approved the final manuscript.

\section{Acknowledgements}

The first author would like to thank her mother and father for raising her to see the world.

\section{References}

1. Nellemann C, Corcoran E, Duarte CM, Valdés L, De Young, et al. Blue Carbon. A rapid response assessment. United Nations Environment Programme, GRID-Arendal, 2009; http://www. grida. no

2. Duarte CM, Middelburg JJ, and Caraco N. Major role of marine vegetation on the oceanic carbon cycle. Biogeosciences. 2005; 1: 17380.

3. Muraoka D. Seaweed resources as a source of carbon fixation. Bull Fish Res Agen Supplement. 2004;1:59-63.

4. Chung IK, Beardall J, Mehta S, Sahoo D, Stojkovic S. Using marine macroalgae for carbon sequestration: a critical appraisal. J Appl Phycol. 2011; 23:877-86.

5. Kitade Y, Taguchi G, Shin JA, Saga N. Porphyra monospore system (Bangiales, Rhodophyta): a model for the developmental biology of marine. plants. Phycol Res. 1998; 46:17-20.

6. Saga N, Kitade Y. Porphyra: a model plant in marine sciences, Fish. Sci. 2002; 68(Suppl):1075-8.

7. Zou DH, Gao KS. Photosynthetic bicarbonate utilization in Porphyra haitanensis (Bangiales, Rhodophyta). Chinese Science Bulletin. 2002; 47:1629-33.

8. Moulin P, Andria JR, Axelsson L, Mercado JM. Different mechanism of inorganic carbon acquisition in red macroalgae (Rhodophyta) revealed by the use of TRIS buffer. Aquatic Botany. 2011; 95: 31-8.

9. Zhang BY, Xie XJ, Liu XH, He LW, Sun YY, Wang GC. The carbonate concentration mechanism of Pyropia yezoensis (Rhodophyta): evidence from transcriptomics and biochemical data. BMC Plant Biology. 2020; 20: 424. https://doi.org/10.1186/s12870-020-02629-4.

10. Badger MR and Price GD. The Role of Carbonic Anhydrase in Photosynthesis. Annual Review of Plant Physiology and Plant Molecular Biology.1994; 45(1):369-92.

11. Karlsson J, Clarke AK, Chen ZY, Hugghins SY, Park YII, et al. A novel alpha -type carbonic anhydrase associated with the thylakoid membrane in Chlamydomonas reinhardtii is required for growth at ambient $\mathrm{CO}_{2}$. The EMBO Journal. 1998;17(5):1208-16.

12. Osborn HL, Alonso-Cantabrana H, Sharwood RE, Covshoff S, Evans JR, Furbank RT. et al. Effects of reduced carbonic anhydrase activity on $\mathrm{CO}_{2}$ assimilation rates in Setaria viridis: a transgenic analysis. Journal of Experimental Botany. 2017; 68(2): $299-310$.

13. So AK, Espie GS, Williams EB, Shively JM, Heinhorst S, Cannon GC. A novel evolutionary lineage of carbonic anhydrase ( $\varepsilon$ class) is a component of the carboxysome shell. J Bacteriol. 2004; 186: 623-30.

14. Moroney JV, Ma YB, Frey WD, Fusilier KA, and Pham TT. The carbonic anhydrase isoforms of Chlamydomonas reinhardtii: intracellular location, expression, and physiological roles. Photosynthesis Research. 2011; 109(1-3):133-49.

15. Kikutani S, Nakajima K, Nagasato C, Tsuji Y, Miyatake Ai, Matsuda Y. Thylakoid luminal $\theta$-carbonic anhydrase critical for growth and photosynthesis in the marine diatom Phaeodactylum tricornutum. Proc. Natl. Acad. Sci. U. S. A. 2016: 113(35)9828-33.

16. Markelova AG, Sinetova MP, Kupriyanova EV, Pronina NA. Distribution and functional role of carbonic anhydrase Cah3 associated with thylakoid membranes in the chloroplast and pyrenoid of Chlamydomonas reinhardtii. Russ J Plant Physiol. 2009; 56(6):761-68.

Page 9/16 
https://doi.org/10.1134/S1021443709060053

17. Tachibana M, Allen AE, Kikutani S, Endo Y, Bowler C. et al. Localization of putative carbonic anhydrases in two marine diatoms, Phaeodactylum tricornutum and Thalassiosira pseudonana. Photosynth Res. 2011; 109:205-21. https://doi.org/10.1007/s11120-0119634-4

18. Ye RX, Yu Z, Shi WW, Gao HJ, Bi YH, Zhou ZG. Characterization of a-type carbonic anhydrase (CA) gene and subcellular localization of aCA in the gametophytes of Saccharina japonica. Journal of Applied Phycology. 2014; 26(2):881-90.

19. Bi YH, Du AY, Li JL, Zhou ZG. Isolation and characterization of a y-carbonic anhydrase localized in the mitochondria of Saccharina japonica. Chemosphere. 2021, 266: 129162. 10.1016/j.chemosphere.2020.129162

20. Zhang BY, Yang F, Wang GC. Cloning and quantitative analysis of the carbonic anhydrase gene from Porphyra yezoensis. J. Phycol., 2010;46 (2):290-96.

21. Wang DM, Yu XZ, Xu KP, Bi GQ, Cao M. et al. Pyropia yezoensis genome reveals diverse mechanisms of carbon acquisition in the intertidal environment. Nature Communications. 2020; 11(1): 4028. Doi: 10.1038/s41467-020-17689-1.

22. Xu K, Chen HZ, Wang WL, Xu Y, Ji DH, Chen CS. et al. Responses of carbonic anhydrases and Rubisco to abrupt CO2 changes of seawater in two marine diatoms. Algal Research. 2017; 28:200-10.

23. Wang YY, Xu K, Wang WL, Xu Y, Ji DH, Chen CS, Xie CT. Physiological differences in photosynthetic inorganic carbon utilizationbetween gametophytes and sporophytes of the economically important red algae Pyropia haitanensis. Algal Research. 2019; 39:101436.

24. Kimber MS. and Pai EF. The active site architecture of Pisum sativum $\beta$-carbonic anhydrase is a mirror image of that of $a$-carbonic anhydrases. EMBO Journal. 2000; 19:1407-18.

25. DiMario RJ, Machingura MC, Waldrop GL, Moroney JV. The many types of carbonic anhydrases in photosynthetic organisms. Plant Science. 2018; 268:11-17.

26. Tachibana, M., Allen, A.E., Kikutani, S. et al. Localization of putative carbonic anhydrases in two marine diatoms, Phaeodactylum tricornutum and Thalassiosira pseudonana. Photosynth Res. 2011; 109:205-21. https://doi.org/10.1007/s11120-011-9634-4.

27. Parisi G, Fornasari M, and Echave J. Evolutionary analysis of gamma-carbonic anhydrase structurally related proteins. Mol. Phylogenet. Evol. 2000; 14:323-34.

28. Iverson TM, Alber BE, Kisker C, Ferry JG. and Rees DC. A closer look at the active site of gamma-class carbonic anhydrases: highresolution crystallographic studies of the carbonic anhydrase from Methanosarcina thermophila. Biochemistry. 2000; 39:9222-31.

29. Candat A, Paszkiewicz G, Neveu M, Gautier R, Logan DC, Avelange-Macherel, MH, Macherel D. The ubiquitous distribution of late embryogenesis abundant proteins across cell compartments in Arabidopsis offers tailored protection against abiotic stress. Plant Cell. 2014; 2:3148-66.

30. DiMario RJ, Clayton H, Mukherjee A, Ludwig M, Moroney JV. Plant carbonic anhydrases: structures, locations, evolution, and physiological roles. Mol. Plant. 2017; 10:30-46.

31. Fabre N, Reiter IM, Becuwe-Linka N, Genty B, Rumeau D. Characterization expression analysis of genes encoding alpha and beta carbonic anhydrases in Arabidopsis, Plant Cell Environ. 2007; 30:617-29.

32. Cardol P, Vanrobaeys F, Devreese B, Van Beeumen J, Matagne RF, Remacle C. Higher plant-like subunit composition of mitochondrial complex I from Chlamydomonas reinhardtii: 31 conserved components among eukaryotes. Biochim. Biophys. Acta. 2004;1658:212-24.

33. Cardol P, Gonzalez-Halphen D, Reyes-Prieto A, Baurain D, Matagne RF, Remacle C. The mitochondrial oxidative phosphorylation proteome of Chlamydomonas reinhardtii deduced from the genome sequencing project. Plant Physiol. 2005; 137: 447-59.

34. Fromm S, Braun H-P. and Peterhänsel C. Mitochondrial gamma carbonic anhydrases are required for complex I assembly and plant reproductive development. New Phytologist 2016a; 211:194-207 doi: 10.1111/nph.13886.

35. Fromm S, Senklera J., Zabaletac E., Peterhänsel C. and Braun H-P. The carbonic anhydrase domain of plant mitochondrial complex I. Physiologia Plantarum. 2016b; 157:289-96. doi:10.1111/ppl.12424.

36. Teixeira PF. and Glaser E. Processing peptidases in mitochondria and chloroplasts. Biochim. Biophys. Acta. 2013;1833:360-70.

37. Avelange-Macherel M-H, Candat A, Neveu M, Tolleter D and Macherel D. Decoding the divergent subcellular location of two highly similar paralogous LEA protein. International Journal of Molecular Sciences. 2018; 19:1620. 10.3390/ijms19061620

38. DiMario RJ, Quebedeaux JC, Longstreth DJ, Dassanayake M, Hartmann MM, Moroney JV. The cytoplasmic carbonic anhydrases beta CA2 and beta CA4 are required for optimal plant growth at low CO2, Plant Physiol. 2016; 171: 280-93.

39. Cousins AB, Badger MR, Caemmerer SV. Carbonic anhydrase and its influence on carbon isotope discrimination during C4 photosynthesis. Insights from antisense RNA in Flaveria bidentis, Plant Physiology. 2006; 141:232-42. www.plantphysiol.org 
40. Li XS, Xu JT, He PM. Comparative research on inorganic carbon acquisition by the macroalgae Ulva prolifera (Chlorophyta) and Pyropia yezoensis (Rhodophyta). J Appl Phycol. 2016; 28:491-97.

41. Nikolau, BJ, Ohlrogge, J.B.; Wurtele, E.S. Plant biotin-containing carboxylases. Arch. Biochem. Biophys. 2003; 414:211-22.

42. Tong L. Structure and function of biotin-dependent carboxylases. Cell Mol. Life Sci. 2013, 70, 863-91

43. He LW, Huang AY, Niu JF, Lu XP, Wang GC. Photosynthetic and metabolic analyses reveal a higher resistance to salinity in Pyropia yezoensis (Rhodophyta) blades than in the conchocelis. Algal research. 2019; 20:101483.

44. Wei L, Wang QT, Yang J, Hu HH and Xu J. RNAi-based targeted gene knockdown in the model oleaginous microalgae Nannochloropsis oceanica. The Plant Journal. 2017, 89:1236-50.

45. Thompson JD, Gbson TJ, Higgins DG. UNIT2.3 multiple sequence alignment using clustal W and Clustal X. Current protocols in Bioinformatics. 2002; DOI: 10.1002/0471250953.bi0203s00

46. Tamura K, Stecher G, Peterson D, Filipski A and Kumar S. MEGA6: Molecular Evolutionary Genetics Analysis version 6.0. Molecular Biology and Evolution. 2013; 30(12): 2725-29. https://doi.org/10.1093/molbev/mst197

47. Heazlewood JL, Tonti-Filippini JS, Gout AM, Day DA, Whelan J, and Millar AH. Experimental analysis of the Arabidopsis mitochondrial proteome highlights signaling and regulatory components, provides assessment of targeting prediction programs, and indicates plantspecific mitochondrial proteins. The Plant Cell. 2004; (16): 241-56.

48. Lee DW and Hwang I. Transient expression and analysis of chloroplast proteins in Arabidopsis protoplasts. Methods Mol Biol. 2011; 774:59-71.

\section{Figures}




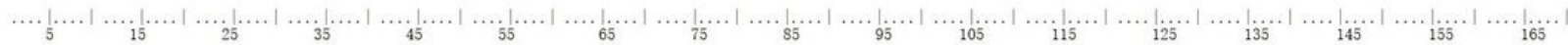

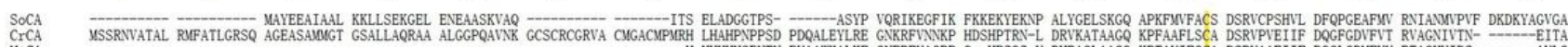

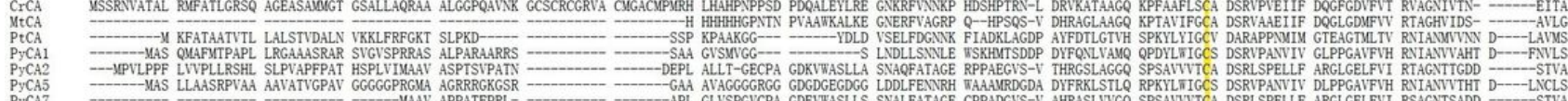

PYCA5

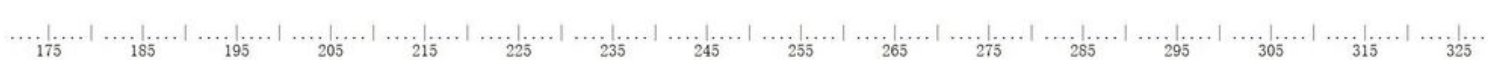

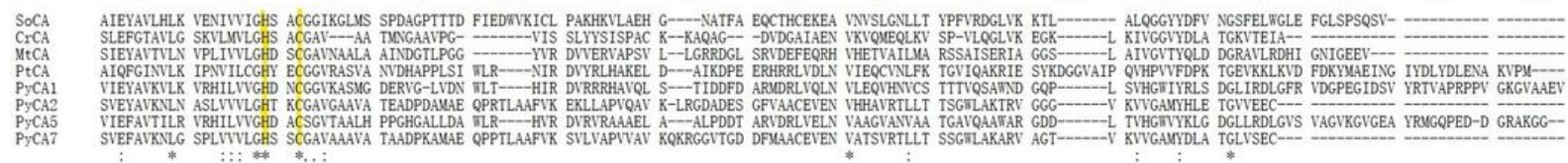

DSCA AHVWMASQND GSDQLAVIGI WYKYGEEDPF LKRLQETAQS NGEAADKNVE LNSFSINVAR DLLPESDLTY YGYDGSLTTP GCDERVKWHV FKEARTVSVA QLKVFSEVTL AAHPEATVTN NRVIQPLNGR KVYEYKGEPN DKYNYYQHGF DWRDNGLDSC AGDVQSPIDI VTSTLQAGSS 360 CrCA
NgCA NgCA
ATCA

AtCA
PyCA3
PyCA4

PyCA6

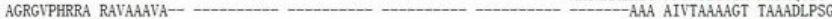
-AIEFFGPRG PAFWGAVS-- PTCA TGLAQSPIDV VATAPAGPLS

DSCA RSDUSSINLN DLNTDAFTLT GNTVIGQGN QINFGDPPAG DLPVIRIGTR DVTFRPLQVH MHFFLSEATV DGVHYPLEAH IVYKDNDNLG DSAGQLAVIG IMYKYG--DA DPFITDUQKR VSDKIASGAI TYGQS---G VSLNNPDDPF NVNIKINFLP SELGYAGYDG SLTTPPCSEI 540

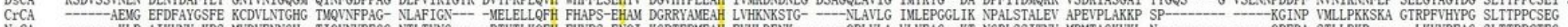

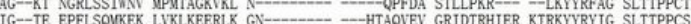

DSCA VKRHVFLEPR TVSVEQMEVF ADVTLNSNPG ATVTTNRMIQ PLEGRTVYGY NGAAA-- - 610

CrCA VDIFVFYQPI KVPDSQILDF XRFYGDNK-- TYATNTRPLQ LLNSRLVEYE LAA -

ATCA VSWTILGKVR SMSKEQVELL RSPLDTS--- -FKNNSRPCQ PLNGRRVEYFF HDHERVDKKE TGNKKKKP

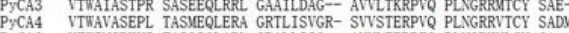

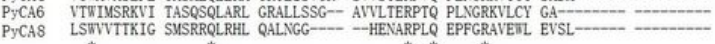

$c$

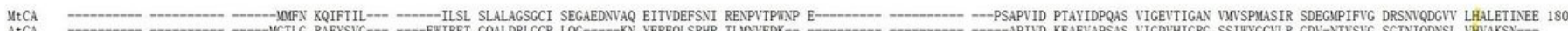

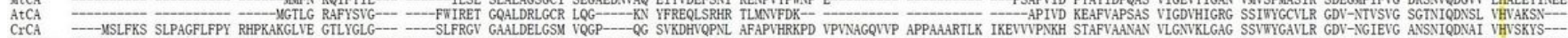

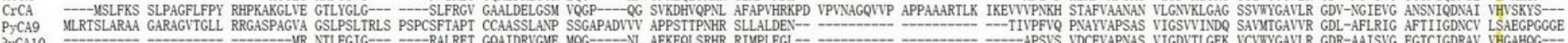

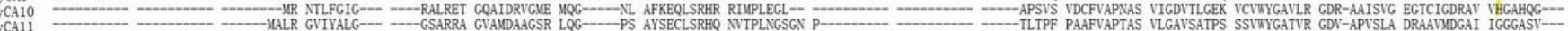

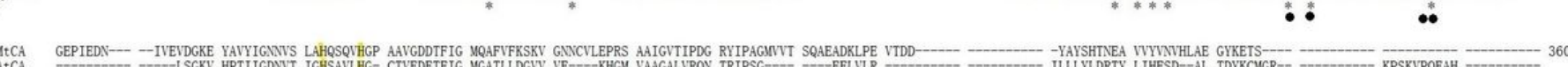

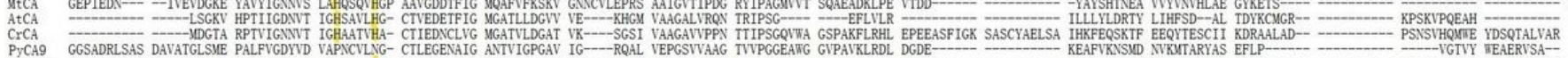

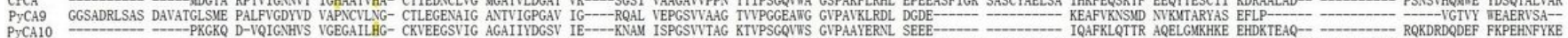
PyCA10
PyCAII

XtCA -370

ATCA

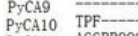

PYCA11 AGGRRQQC

\section{Figure 1}

Alignment of the full-length ORFs of PyCAs and other previously reported CA proteins. A: $\beta C A$ alignment, B: aCA alignment, C: $y C A s$ alignment. *, completely conserved amino acid; zinc coordination residues are shown in yellow. Black bullets indicate essential residues predicted to play essential roles in YCAs. SoCA, CA from Spinacia oleracea; CrCA, CA from Chlamydomonas reinhardtii; MtCA, CA from Mycobacterium tuberculosis H37Rv; PtCA, CA from Phaeodactylum tricornutum. 


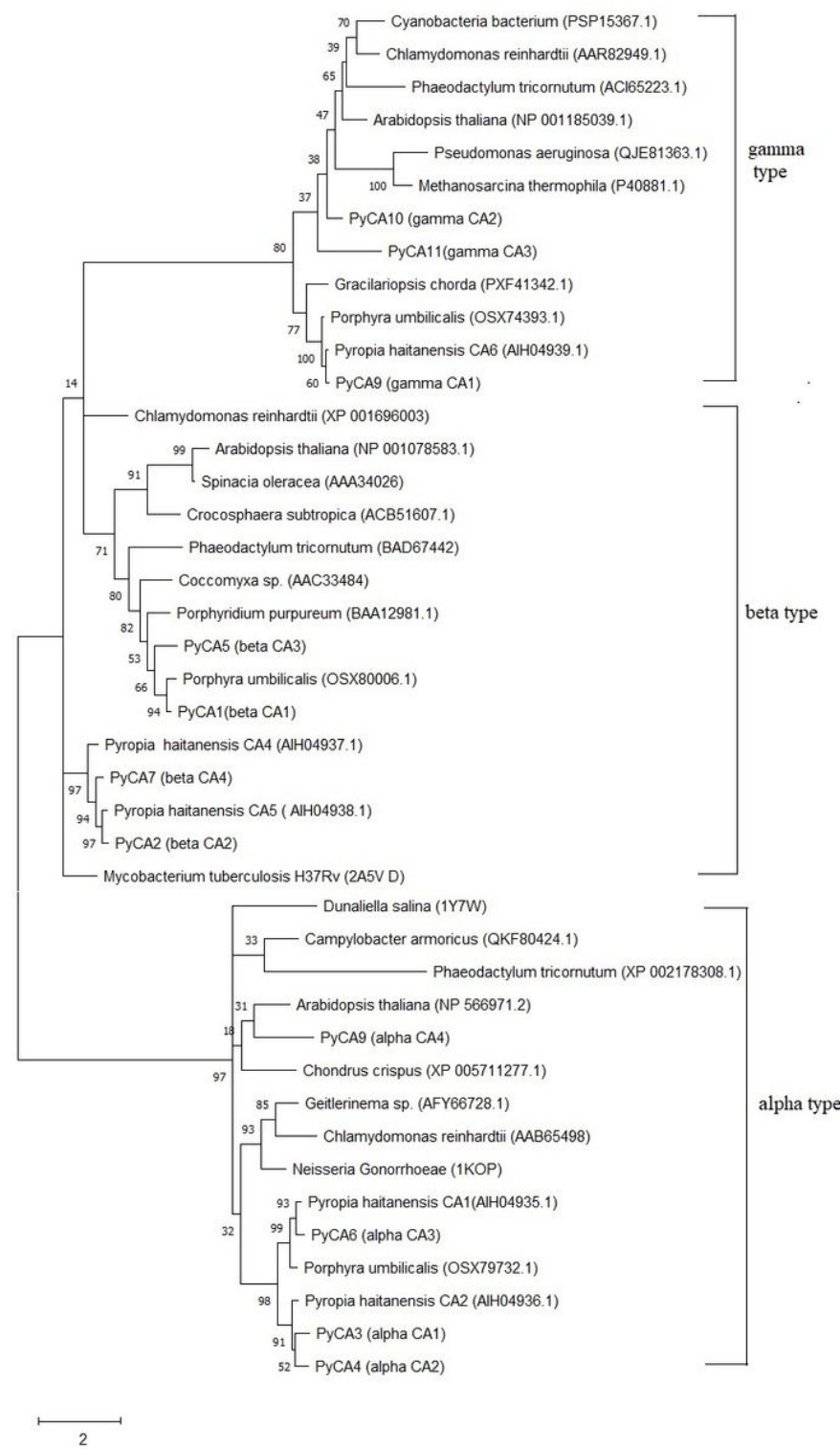

\section{Figure 2}

Unrooted phylogenetic tree of CA proteins. Putative CAs were aligned with ClustalW and analyzed using MEGA version 6 software and the maximum-likelihood method. Accession numbers are shown in parentheses. 

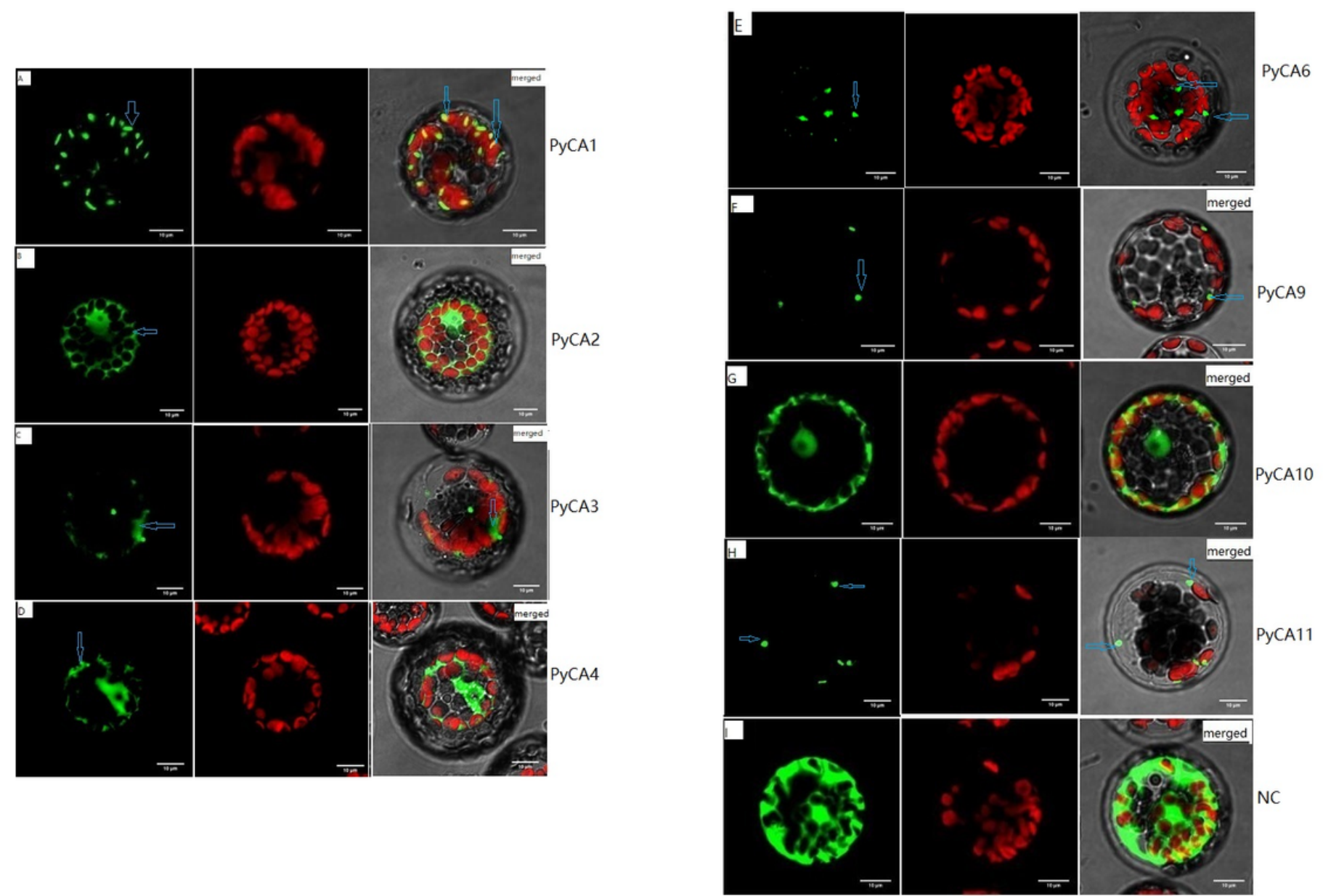

Figure 3

Transient expression of PyCA-GLosgfp protein in Arabidopsis protoplasts. Confocal microscopy images of fusion proteins located in different compartments in transgenic Arabidopsis protoplasts. A-H: PyCA1:GLosgfp, PyCA2:GLosgfp, PyCA3:GLosgfp, PyCA4:GLosgfp, PyCA6:GLosgfp, PyCA9:GLosgfp, PyCA10:GLosgfp and PyCA11:GLosgfp. I: pBWA(V)HS-GLosgfp empty plasmid. Green, GFP; red, chlorophyll. Images from left to right represent green fluorescence from the target gene, chlorophyll fluorescence, and the merged image. Blue arrows show the subcellular localization of PyCA:GFP fusion protein.

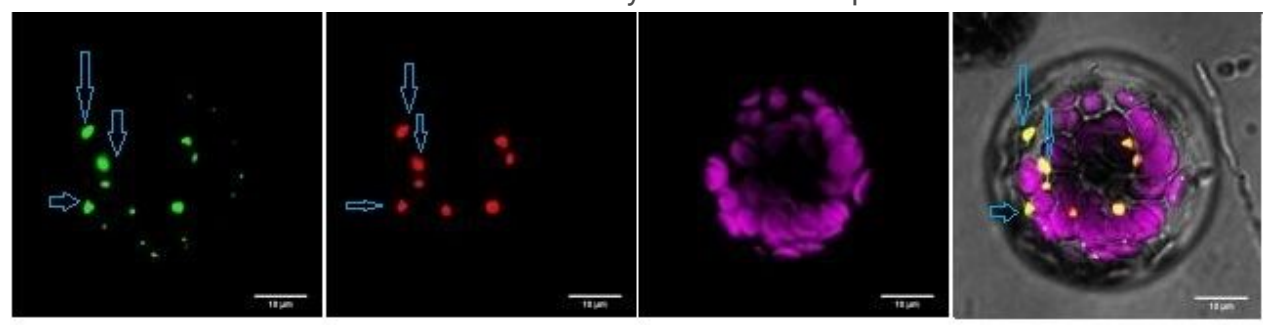

\section{Figure 4}

Redetermining the localization of PyCA6 in Arabidopsis protoplast. Green, red, pink and yellow signals are PyCA6:gfp, MSTP (mitochondrionspecific strain), chlorophyll fluorescence and merged fluorescence from GFP and MKATE, respectively. Blue arrows show the subcellular localization of PyCA6:mkate fusion protein. 

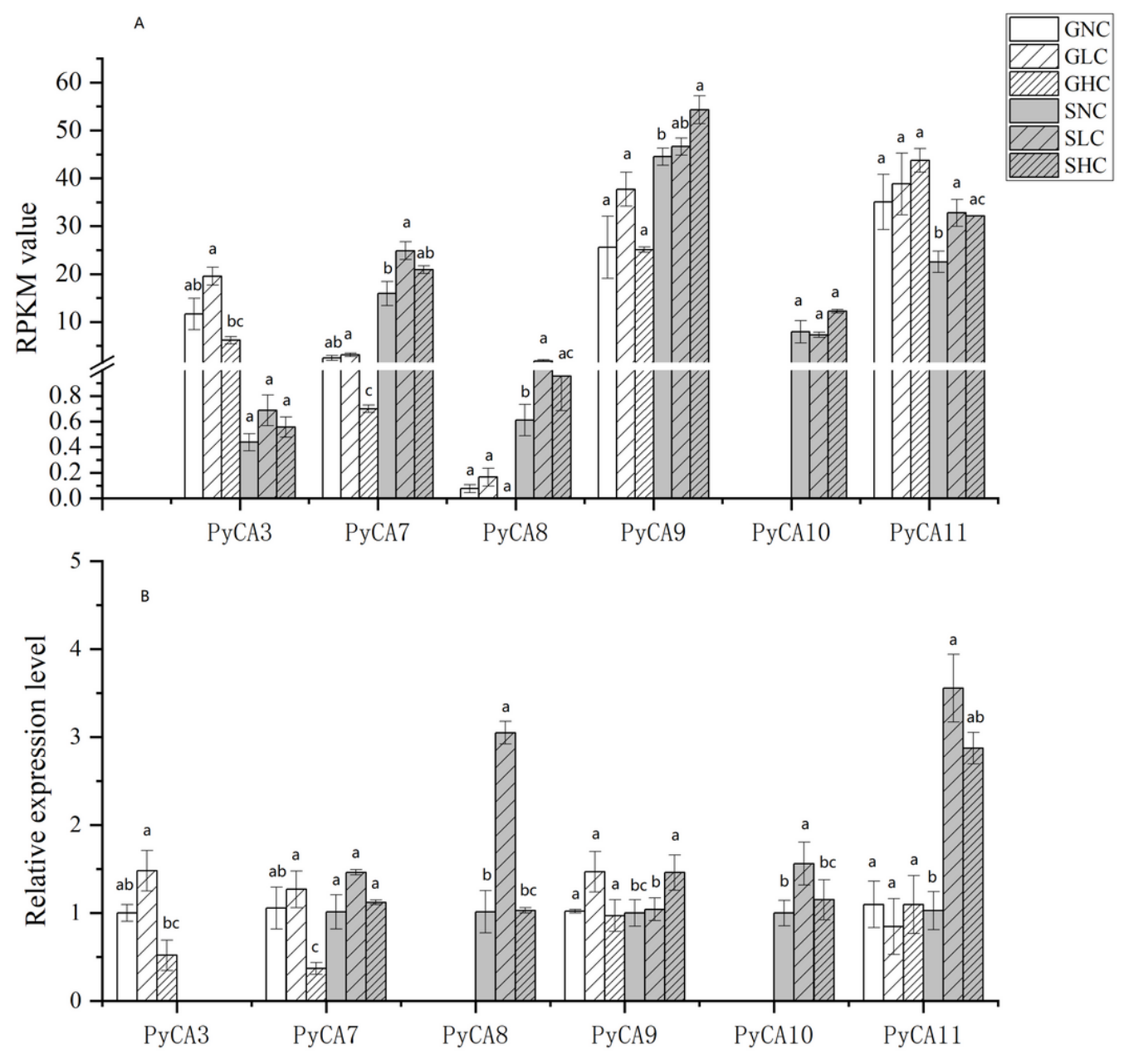

\section{Figure 5}

Expression levels of PyCA3, PyCA7-11 genes based on RNA-Seq assays (A) and relative expression levels verified by qRT-PCR (B) in gametophytes and sporophytes of $\mathrm{P}$. yezoensis under three $\mathrm{Ci}$ conditions. Data represent the mean \pm standard deviation from three biological replicates. Means followed by different lowercase letters are significantly different between different Ci treatments $(\mathrm{p}<0.05)$. 


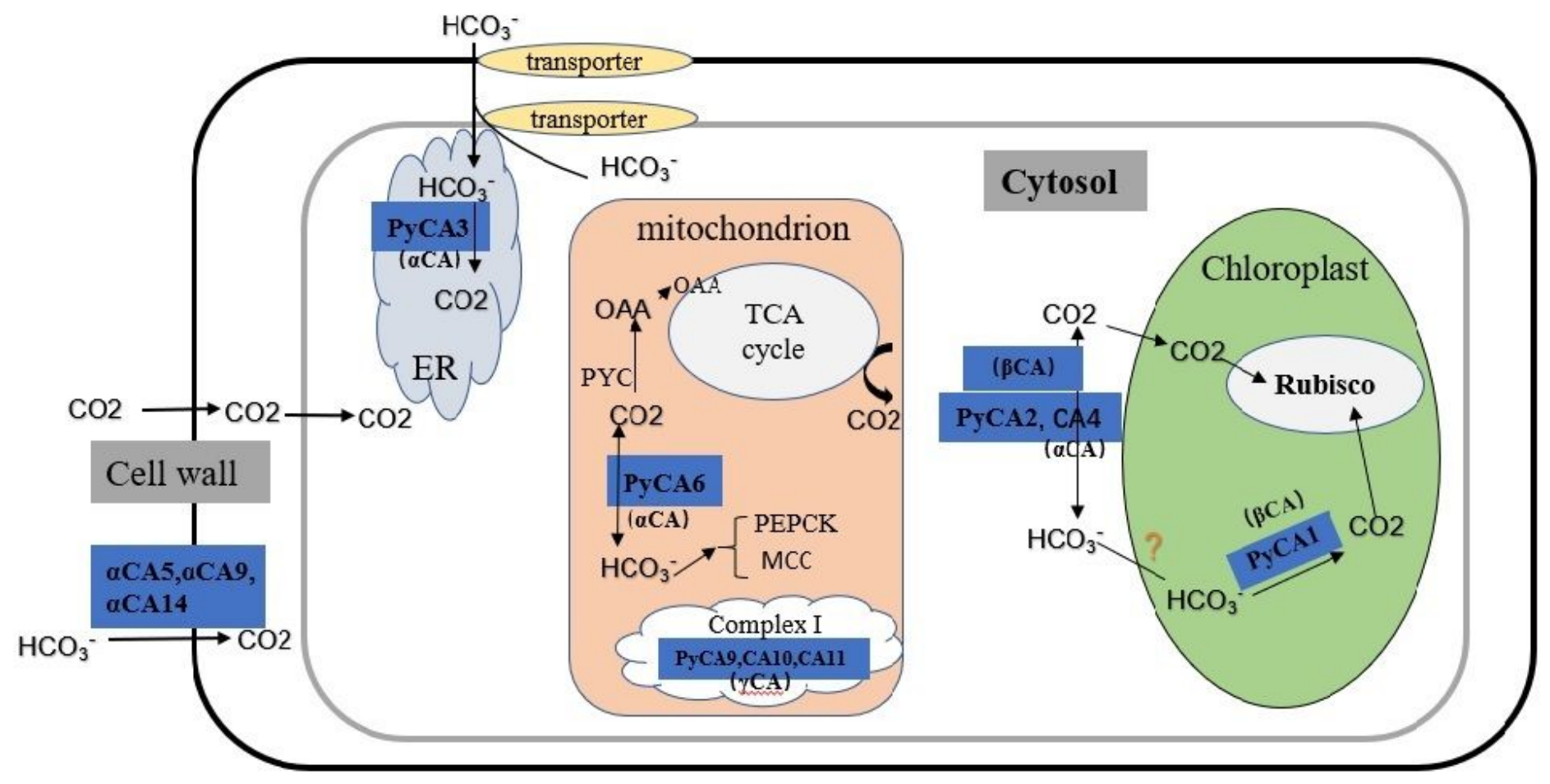

Figure 6

Schematic diagram of PyCA localization and putative inorganic carbon utilization styles in P. yezoensis. ER: endoplasmic reticulum; Rubisco: ribulose-1,5-bisphosphate carboxylase/oxygenase; PYC: pyruvate carboxylase; OAA: oxaloacetate; PEPCK: phosphoenolpyruvate carboxykinase; MCC: 3-methylcrotonyl-CoA carboxylase.

\section{Supplementary Files}

This is a list of supplementary files associated with this preprint. Click to download.

- S1Tables1.docx

- S2Fig1exonandintronstructure.jpg

- S314CAmatrix.jpg

- S4TableS2.docx

- S5TableS3.docx 